Check for updates

Cite this: Phys. Chem. Chem. Phys. 2021, 23, 23922

Received 25th July 2021,

Accepted 6th September 2021

DOI: $10.1039 / d 1 c p 03405 g$

rsc.li/pccp

\section{THz/Far infrared synchrotron observations of superlattice frequencies in $\mathrm{MgB}_{2} \dagger$}

\author{
Jose A. Alarco, (D) abc Bharati Gupta, abc Mahboobeh Shahbazi, (iD) abc \\ Dominique Appadoo and Ian D. R. Mackinnon (D) *ae
}

\begin{abstract}
$\mathrm{THz} /$ Far Infrared synchrotron absorption experiments on pure and doped $\mathrm{MgB}_{2}$ samples show that the absorption spectral weight at low wavenumber (i.e., $<110 \mathrm{~cm}^{-1}$ ) evolves as the temperature is reduced to $10 \mathrm{~K}$. Distinct spectral peak intensities increase as the temperature of $\mathrm{MgB}_{2}$ and doped $\mathrm{MgB}_{2}$ approaches, and then crosses, the superconducting transition temperature. These experimental data suggest a strong link to superconductivity induced by subtle shifts in structural symmetry. Significant increases in absorption are observed at frequencies that correspond to the superconducting gaps for doped and pure $\mathrm{MgB}_{2}$, and at fractions of these frequency (or energy) values. This low wavenumber spectral transition is consistent with the notion that superlattice frequencies contribute to the optic modes of the $\mathrm{MgB}_{2}$ phonon dispersion and are critical to the superconducting transition for this structure. Key integer ratios are identified in real and reciprocal spaces that link bonding character, Fermi vectors and Fermi surfaces as well as phonon properties with geometric parameters and specific superlattice symmetries for $\mathrm{MgB}_{2}$. Similarly consistent spectral data at low wavenumber are also obtained for carbon doped $\mathrm{Mg}^{11} \mathrm{~B}_{2}$. Density Functional Theory calculations of superlattice phonon dispersions result in folded mode frequencies that match these observed low wavenumber experiments. These results show that symmetry reductions, largely electronic in character although coupled to vibrations, occur with change in temperature and imply strong links to superconductivity mechanisms.
\end{abstract}

\section{Introduction}

Measurement and analyses of acoustic waves have played a major role in the understanding of superconductivity, particularly for pure elemental metals and ordered intermetallic compounds. ${ }^{1}$ Acoustic phonons have been key to formulation of concepts for BCS superconductivity, ${ }^{2,3}$ explanation of isotopic effects $^{4-6}$ and for other manifestations of superconductivity. ${ }^{1}$ For example, anomalies in the acoustic phonon dispersions (PDs) of A15 superconducting materials have been correlated with their relatively high superconducting transition temperature $\left(T_{\mathrm{c}}\right)$, and attributed to anharmonic effects and/or phonon mode softening. ${ }^{1}$

\footnotetext{
${ }^{a}$ Centre for Clean Energy Technologies and Practices, Faculty of Science, Queensland University of Technology, Brisbane, QLD 4001, Australia. E-mail: ian.mackinnon@qut.edu.au

${ }^{b}$ Centre for Materials Science, Faculty of Science, Queensland University of Technology, Brisbane, QLD 4001, Australia

${ }^{c}$ School of Chemistry and Physics, Faculty of Science, Queensland University of Technology, Brisbane, QLD 4001, Australia

${ }^{d}$ Australian Synchrotron, Clayton, VIC, Australia

${ }^{e}$ School of Earth and Atmospheric Sciences, Faculty of Science, Queensland University of Technology, Brisbane, QLD 4001, Australia

$\dagger$ Electronic supplementary information (ESI) available. See DOI: 10.1039/ d1cp03405g
}

While acoustic anomalies have also been partly addressed in higher temperature superconductors of quite different composition, ${ }^{7}$ attention in recent years has largely shifted to optical phonons, due to evident correlations with electronic band structures and to correspondence of PDs at higher frequencies to optic modes. ${ }^{1}$ $\mathrm{MgB}_{2}$ is a representative example of this trend, where the optical $\mathrm{E}_{2 \mathrm{~g}}$ mode has been extensively implicated in superconductivity. ${ }^{8,9}$ Furthermore, significant correlations between PD anomalies and the superconducting gap energy have been identified for $\mathrm{MgB}_{2}$ for a wide range of external conditions. ${ }^{10-12}$

Terahertz $\left(\mathrm{TH}_{\mathrm{Z}}\right)$ or Far Infrared refers to radiation that covers between the microwave and infrared (IR) regions of the electromagnetic spectrum ${ }^{13}\left(1 \mathrm{THz}=4.136 \mathrm{meV}=33.356 \mathrm{~cm}^{-1}\right)$. In synchrotrons, where such radiation is generated by electrons moving at relativistic speeds, ${ }^{14} \mathrm{THz}$ radiation or light is highly intense and collimated, so that it finds applications in many fields of research, such as nanoimaging and nano-spectroscopy. ${ }^{15}$ At energies $<400 \mathrm{~cm}^{-1}$, synchrotron radiation flux outperforms conventional thermal sources for data fidelity, making the THz/Far IR region of particular interest for low energy phenomena. ${ }^{16,17}$ The meV and sub-meV range of $\mathrm{THz}$ energy involves the acoustic regions of PDs and the typical energy of superconducting gaps. Previous synchrotron $\mathrm{THz}$ experiments on superconductors have been carried out on boron-doped diamond, $\mathrm{V}_{3} \mathrm{Si}$ 
(an A15-type intermetallic), $\mathrm{MgB}_{2}$ and isostructural CaAlSi using reflection and transmission modes. ${ }^{17,18}$ These experiments generally observed broad absorption features that appear to have a temperature dependence correlated with respective $T_{\mathrm{c}} \mathrm{S}$ or gap energies.

The THz/Far IR region is also ideal for investigation of superlattice frequencies which are generally located in the low wavenumber range. We have previously observed indications of superlattice periodicities in Raman spectra of $\mathrm{MgB}_{2}{ }^{19}$ and $\mathrm{YB}_{6}{ }^{20}$ superconductors. Using Density Functional Theory (DFT) to calculate PDs ${ }^{21}$ and superlattice constructs, which fold the reciprocal space of the original crystal, enables identification of new lower frequency peaks activated for Raman detection. These calculations give a remarkable match to observed small peaks ${ }^{19}$ at the limit of laboratory detection. Other work by Campi et $a .^{22}$ implies that a $4 \times$ superlattice along the $c$-axis direction may have been detected in $\left(\mathrm{Mg}_{1-x} \mathrm{Al}_{x}\right) \mathrm{B}_{2}$ by high resolution neutron diffraction.

This article describes detection of low wavenumber signals from $\mathrm{MgB}_{2}$ and $\mathrm{C}$-doped $\mathrm{MgB}_{2}$ superconductors at and below the $T_{\mathrm{c}}$ value. These distinctive changes in spectral intensity occur within the acoustic range of frequencies for the accepted $P 6 / \mathrm{mmm}$ high symmetry of $\mathrm{MgB}_{2}$ and are less evident or absent at higher temperatures (e.g., $>100 \mathrm{~K}$ ). We utilise DFT computation to identify potential superlattice constructs for $\mathrm{MgB}_{2}$ that explain these low frequency observations.

\section{Experimental}

Pure $\mathrm{MgB}_{2}$ powders, one synthesized in-house and another outsourced from Sigma Aldrich, as well as a sample of $10 \%$ carbon doped $\mathrm{Mg}^{11} \mathrm{~B}_{2}$ powder (made in-house with $10 \% \mathrm{C} 400$ and boron ${ }^{11} \mathrm{~B}$ isotope) were used for THz/Far IR analysis. The superconducting transitions for these $\mathrm{MgB}_{2}$ samples are shown in Fig. S1 (ESI $\dagger$ ). The lattice parameters, transition temperature $\left(T_{\mathrm{c}}\right)$ and cell volume for carbon doped $\mathrm{Mg}^{11} \mathrm{~B}_{2}$ decreased after carbon doping compared to those for pure $\mathrm{Mg}^{11} \mathrm{~B}_{2}$. Lattice parameters $(a=3.07534(5) \AA$ and $c=3.52318(9) \AA)$ and cell volume (28.857(1) $\AA^{3}$ ) values obtained using Rietveld refinement as well as $T_{\mathrm{c}}$ are consistent with previous studies of carbon doped $\mathrm{MgB}_{2} \cdot{ }^{23}$ Powders of each sample were thoroughly mixed with polyethylene (PE) powder using a mortar and pestle inside a nitrogen filled glove box, and pressed into small $3 \mathrm{~mm}$ diameter, hollow stainless disks, included in a die set from PIKE. ${ }^{25}$

Fine-grained $\mathrm{MgB}_{2}$ is a black powder and so absorbs strongly in the IR region. Because the expected level of absorption from samples under experimental conditions was unknown, a wide range of $\mathrm{MgB}_{2}: \mathrm{PE}$ mixtures were prepared for preliminary analysis. Ratios of $\mathrm{MgB}_{2}: \mathrm{PE}$ between $70: 30$, $50: 50,35: 65,15: 85,5: 95,4: 96$ and $3: 97$ were initially scanned at room temperature to determine optimum ratios for transmission of a signal. Pellets with high ratios of $\mathrm{MgB}_{2}$ did not provide clear transmittance signals; consequently, subsequent work focused on pellets with $4 \%$ and $5 \% \mathrm{MgB}_{2}$ content.
Images of the He-cryostat and the insertable sample holder for transmission measurements, which are later converted to absorption values, are shown in ESI $\dagger$ (Fig. S2). The THz/Far IR beamline is equipped with a Bruker IFS 125/HR Fourier transform (FT) spectrometer, and for this work, the spectrometer is coupled to a $6 \mathrm{~K}$ closed loop pulse-tube (CLPT) cryostat from Cryo-Industries of America. The spectrometer is fitted with a $6 \mu \mathrm{m}$ multilayer Mylar beam splitter and a Si Bolometer from Infrared Laboratories Ltd equipped with a $13 \mu \mathrm{m}$ cut-on longpass cold filter to record the transmittance spectrum between 20-650 $\mathrm{cm}^{-1}$. Spectra are collected at specific temperatures in the range $6 \mathrm{~K}$ to $296 \mathrm{~K}$; five separate spectra are collected at each temperature and averaged to improve quality of signal to noise ratios. The OPUS 8.0 software is used for data acquisition and analysis. To deconvolute spectra from sample-PE composites, absorption results for each composite are divided by measurements at the same temperature for PE only under the same conditions.

\section{Calculation procedures}

Electronic Band Structure (EBS) and PD calculations are undertaken using Density Functional Theory (DFT) as implemented in the Cambridge Serial Total Energy Package (CASTEP) of Materials Studio (MS) 2017 and 2018. ${ }^{21}$ All structures are optimized for geometry, including cell parameters, starting from the $\mathrm{MgB}_{2}$ crystal information file (.cif) for $P 6 / \mathrm{mmm}$ group symmetry (No. 191), available in standard databases. Crystal structure visualizations, schematics and bond lengths were generated using Crystal-Maker $\mathrm{X}$ version 10.5.3 software based on the $\mathrm{MgB}_{2}$.cif file.

Superlattices in the $c$ axis direction are constructed by multiplying the unit cell along the appropriate direction and imposing reduced symmetries to suit the superlattice construction, in line with procedures described in our earlier publications. ${ }^{19,20,26}$ For higher dimension superlattices (e.g., 4c), where a large computational resource is required for all reciprocal directions, calculations are limited to key, high symmetry reciprocal space directions such as $\Gamma A, \Gamma K$ and $\Gamma M$. In general, the local density approximation (LDA) and generalized gradient approximation (GGA), with norm-conserving pseudopotentials, are used in separate DFT calculations to provide a measure of computational fidelity. ${ }^{11}$

The typical setup for calculations uses a $\Delta k$ value ranging between 0.01 to $0.02 \AA^{-1}$ (or less; i.e., a grid mesh ranging from $45 \times 45 \times 30$ to $19 \times 19 \times 14),{ }^{26}$ with a plane wave basis set cut-off of $990 \mathrm{eV}$, an ultra-fine (or better) customized setup to ensure total energy convergence of less than $5 \times 10^{-6} \mathrm{eV}$ per atom, a maximum force of less than $0.01 \mathrm{eV} \AA^{-1}$, a maximum stress of less than $0.02 \mathrm{GPa}$ and maximum displacements of less than $5 \times 10^{-4} \AA$. These calculation procedures are similar to those previously reported for $\mathrm{MgB}_{2}$ and metal-substituted derivatives of $\mathrm{MgB}_{2}\left(\right.$ e.g.,$\left.\left(\mathrm{Mg}_{1-x} \mathrm{M}_{x}\right) \mathrm{B}_{2}\right)$ where $(\mathrm{M}=\mathrm{Al}$, Ti, Sc, $\mathrm{Cd}, \mathrm{Ba}) .^{10,11,28}$ In general, phonon calculations use a linear approximation method except as annotated where a finite displacement method is used. 


\section{Results}

Results for $\mathrm{THz} /$ Far IR absorption measurements on $\mathrm{MgB}_{2}$ diluted with PE are an important guide to subsequent superlattice calculations which are computationally demanding. Interpretations of these experimental data are supported by results from DFT calculations for a single $\mathrm{MgB}_{2}$ unit cell (with $P 6 / \mathrm{mmm}$ symmetry) and for an $\mathrm{MgB}_{2}$ superlattice with a multiple of the $c$-lattice parameter.

\section{Absorption spectra in the THz region}

The absorption spectra for $\mathrm{Mg}^{11} \mathrm{~B}_{2}$ doped with carbon (10\%) and diluted with $\mathrm{PE}\left(\mathrm{Mg}^{11} \mathrm{~B}_{2}\right.$ : $\mathrm{PE}$ ratio 5:95) with minimal processing of spectral data is shown in Fig. 1a. Each spectrum at a specific temperature has been offset vertically for visual clarity. New spectral features occur in the low wavenumber region as the sample temperature approaches $40 \mathrm{~K}$, a few degrees above the superconducting transition. In this case, the onset $T_{\mathrm{c}}$ for $\operatorname{Mg}\left({ }^{11} \mathrm{~B}_{0.9} \mathrm{C}_{0.1}\right)_{2}$ is measured at $37.1 \mathrm{~K}$ using resistive transition and vibration sample magnetometry (VSM) measurements. Details of onset, mid-point and zero resistance $T_{\mathrm{c}}$ values for both samples are provided in Table S1 (ESI $\dagger$ ).
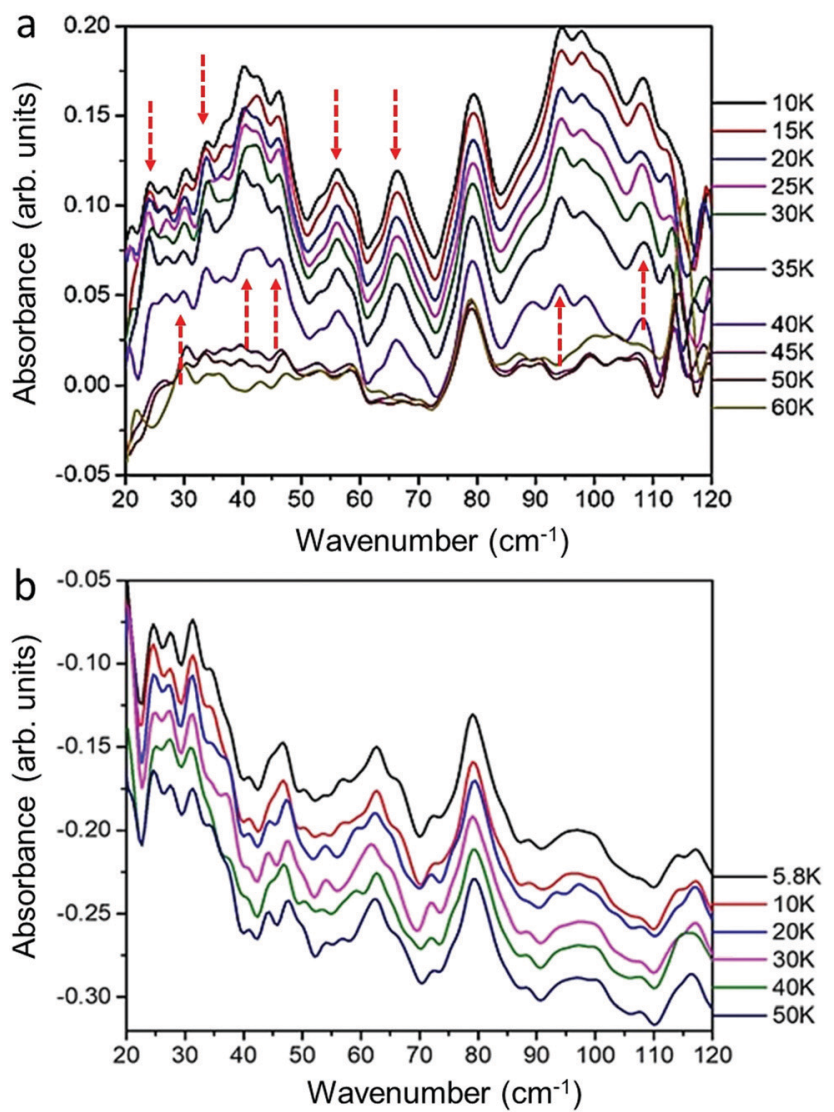

Fig. $1 \mathrm{THz} /$ Far IR absorbance results for (a) composite of $\mathrm{Mg}^{11} \mathrm{~B}_{2}$ with $10 \%$ $\mathrm{C}$ doping diluted with $\mathrm{PE}\left(\mathrm{Mg}^{11} \mathrm{~B}_{2}\right.$ : $\mathrm{PE}$ ratio $\left.5: 95\right)$. Note the clear evolution of spectral density at low wavenumbers as the temperature crosses $T_{\mathrm{c}}$. Key changes to absorbance below $45 \mathrm{~K}$ are highlighted with red dashed arrows and (b) PE prepared by the same method as for $\mathrm{Mg}^{11} \mathrm{~B}_{2}$.
Key changes in spectral features between $20 \mathrm{~cm}^{-1}$ and $120 \mathrm{~cm}^{-1}$ are highlighted with dotted red arrows in Fig. 1a. The substantial increase in low wavenumber spectral intensity as well as the appearance of new low frequency peaks at or below $40 \mathrm{~K}$ suggests a strong connection to superconductivity. Furthermore, these peaks do not diminish at temperatures below $40 \mathrm{~K}$. As is evident in Fig. 1a, the low wavenumber peaks show strong signal to noise and at similar frequencies at temperatures below $T_{\mathrm{c}}$. For comparison, Fig. 1b shows the absorption spectra for PE powder obtained under the same temperature and wavenumber ranges and operating conditions as for C-doped $\mathrm{Mg}^{11} \mathrm{~B}_{2}$ shown in Fig. 1a.

There is a difference in the intensity of peaks obtained on $\mathrm{MgB}_{2}$ and on C-doped $\mathrm{Mg}^{11} \mathrm{~B}_{2}$ at the same temperature and with the same proportions of intermixed PE. The spectra for C-doped $\mathrm{Mg}^{11} \mathrm{~B}_{2}$ display features more distinctive than the spectra for $\mathrm{MgB}_{2}$ with reduction in sample temperature. For comparison, the spectra for $\mathrm{MgB}_{2}$ with $5 \% \mathrm{PE}$ as well as for $\mathrm{C}$-doped $\mathrm{Mg}^{11} \mathrm{~B}_{2}$ with $4 \% \mathrm{PE}$ mixture are shown in ESI $\dagger$ (Fig. S3). In both cases, the presence of stronger peaks for $T<40 \mathrm{~K}$ is evident (dotted red arrows) but the quality and spectral intensity is not as obvious as shown in Fig. 1a. While spectral signal(s) may be influenced by grain orientation and/or preferential texture(s) induced by sample preparation as well as by the quantity of sample diluted with PE, we note below an additional interpretation for this difference in spectral signal.

Table 1 lists deconvolution peak values, grouped in wavenumber segments, for a range of temperatures below and above $T_{\mathrm{c}}$ for C-doped $\mathrm{Mg}^{11} \mathrm{~B}_{2}$ as shown in Fig. 1a. Numbering for peaks shown in Table 1 are identified in ESI $\dagger$ Fig. S4. Estimates of peak intensities shown in Table 1 are normalised to the highest FWHM value within each peak dataset. Detailed peak deconvolution results for a range of temperatures $(10 \mathrm{~K}<T<$ $60 \mathrm{~K}$ in $5 \mathrm{~K}$ increments) are provided in ESI, $\dagger$ as Table S2.

Table 1 lists peaks with relatively high intensities within the wavenumber range $20 \mathrm{~cm}^{-1}$ to $110 \mathrm{~cm}^{-1}$ with change in temperature. Each of these peaks show a consistent wavenumber value between temperatures $60 \mathrm{~K}$ to $6 \mathrm{~K}$ and, similarly, for all peaks in the THz/Far IR spectra shown in ESI $\dagger$ (Fig. S4 and Table S2). For peaks listed in Table 1, the variation in peak position (i.e., wavenumber value) and the standard deviation from the average peak value (for each temperature) is $<0.5 \mathrm{meV}$. Nevertheless, the relative peak intensities vary with temperature between $6 \mathrm{~K}$ and $60 \mathrm{~K}$ as shown in Table 1. For example, the peak at $26.7(3) \mathrm{cm}^{-1}$ shows highest intensity at $45 \mathrm{~K}$, the peak at 79.1(2) $\mathrm{cm}^{-1}$ is highest at $10 \mathrm{~K}$ and $35 \mathrm{~K}$ and the peak at $108.1(3) \mathrm{cm}^{-1}$ is highest at $20 \mathrm{~K}, 30 \mathrm{~K}$ and $60 \mathrm{~K}$. These shifts in relative intensity with temperature reflect subtle shifts in either electron density, atom positions or both, with change in temperature. A comparison of the THz/Far IR spectra and deconvoluted peaks for $30 \mathrm{~K}<$ $T<40 \mathrm{~K}$, along with numeric identification of peaks referenced in Table 1, is provided in the ESI, $\dagger$ section (Fig. S4).

\section{Phonon dispersions - acoustic region}

A PD calculation for the unit cell of $\mathrm{MgB}_{2}$ with $P 6 / \mathrm{mmm}$ symmetry is shown in Fig. 2a. The PD displays key features in 
Table 1 Selected THz/Far IR peak parameters for C-doped $\mathrm{Mg}^{11} \mathrm{~B}_{2}$

\begin{tabular}{|c|c|c|c|c|c|c|c|}
\hline \multirow[t]{6}{*}{ Peak no. } & Temp. [K] & Wave number $\left[\mathrm{cm}^{-1}\right]$ & Relative intensity [Arb.] & Peak no. & Temp. [K] & Wave number $\left[\mathrm{cm}^{-1}\right]$ & Relative intensity [Arb.] \\
\hline & 20 & 26.62 & 48 & \multirow{5}{*}{17} & 20 & 79.23 & 88 \\
\hline & 30 & 26.63 & 60 & & 30 & 79.18 & 97 \\
\hline & 40 & 26.82 & 59 & & 40 & 79.21 & 83 \\
\hline & 45 & 26.10 & 100 & & 45 & 78.95 & 77 \\
\hline & 50 & 27.17 & 35 & & 50 & 79.00 & 63 \\
\hline & 20 & 39.96 & 50 & \multirow{7}{*}{19} & 20 & 90.27 & 95 \\
\hline & 30 & 40.24 & 57 & & 30 & 90.65 & 81 \\
\hline & 35 & 39.86 & 55 & & 35 & 90.15 & 66 \\
\hline & 40 & 40.28 & 59 & & 40 & 90.83 & 59 \\
\hline & 45 & 39.44 & 57 & & 45 & 90.88 & 54 \\
\hline & 50 & 39.61 & 50 & & 50 & 90.92 & 45 \\
\hline & 60 & 39.30 & 39 & & 60 & 91.41 & 59 \\
\hline & 45 & 57.25 & 52 & & 45 & 99.17 & 75 \\
\hline & 50 & 57.15 & 39 & & 50 & 100.37 & 37 \\
\hline & 60 & 57.13 & 37 & & 60 & 100.33 & 72 \\
\hline \multirow[t]{8}{*}{14} & 10 & 66.17 & 77 & \multirow[t]{8}{*}{24} & 10 & 108.16 & 93 \\
\hline & 20 & 66.12 & 70 & & 20 & 107.74 & 100 \\
\hline & 30 & 66.33 & 87 & & 30 & 108.10 & 100 \\
\hline & 35 & 66.26 & 86 & & 35 & 108.50 & 81 \\
\hline & 40 & 66.18 & 71 & & 40 & 108.47 & 67 \\
\hline & 45 & 67.01 & 94 & & 45 & 107.97 & 52 \\
\hline & 50 & 67.52 & 59 & & 50 & 108.04 & 51 \\
\hline & 60 & 67.81 & 67 & & 60 & 107.78 & 100 \\
\hline
\end{tabular}

the optical range including a phonon anomaly associated with the $\mathrm{E}_{2 \mathrm{~g}}$ mode (highlighted in red in Fig. 2a) around $\Gamma$. This phonon anomaly has been previously discussed by many authors and is well correlated with superconductivity. ${ }^{8-11,26}$ We provide vertical and horizontal dashed lines in Fig. 2a as guides to highlight specific features in the acoustic region of the PD (i.e., for wavenumbers less than $\sim 340 \mathrm{~cm}^{-1}$ ).

Note that the lowest optical frequency at the $\Gamma$ point is $\sim 340 \mathrm{~cm}^{-1}$. At this point, absorption of electromagnetic radiation will not take place at wavenumbers below this value because the available phonon states for creation or excitation of optical phonons do not exist. Hence, our focus for these absorption experiments is on the acoustic phonon modes and evidence for their presence in superconductors at or below $T_{\mathrm{c}}$. In Fig. 2a, integer proportions of key reciprocal directions can be identified and are discussed below.

\section{Superlattice calculations - Brillouin zones}

Superlattice calculations in this work follow a similar rationale to that used and discussed in our previous publication on laboratory-based Raman microscopy of $\mathrm{MgB}_{2} \cdot{ }^{19}$ In practice, a reduced symmetry to $P 6 / \mathrm{mmm}$ is assigned so that the full supercell describes all symmetry elements. Calculations are carried out for superlattice periods equivalent to $2 c, 3 c$ and $4 c$ where $c$ is the $c$-axis unit cell dimension of the $P 6 / \mathrm{mmm}$ lattice. These calculations utilise a variety of $k$-grids and LDA and GGA
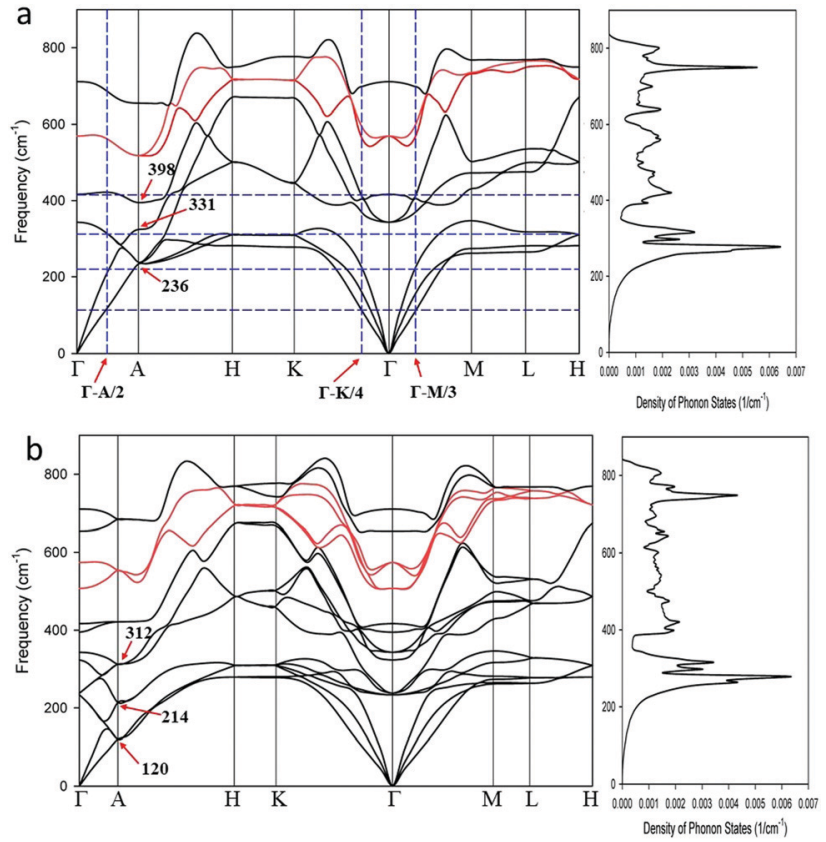

Fig. 2 PD calculated using DFT with the LDA functional $\left(\Delta k=0.01 \AA^{-1}\right)$ for (a) the $\mathrm{MgB}_{2}$ unit cell with $P 6 / \mathrm{mmm}$ symmetry. Dotted lines (blue horizontal and vertical) are guides to identify specific features in the acoustic phonon region along key reciprocal lattice directions. (b) $\mathrm{An} \mathrm{MgB}_{2} 2 \mathrm{C}$ supercell with $P \overline{6} c 2$ symmetry. The optical phonon for the $E_{2 g}$ modes is highlighted in red. Frequency values (in $\mathrm{cm}^{-1}$ ) for acoustic phonons at $A$ are shown. For each case, the phonon density of states is on the right. 
Table 2 DFT PD calculations for $\mathrm{MgB}_{2}$ superlattices along $\mathrm{c}$-axis

\begin{tabular}{|c|c|c|c|c|c|c|c|c|c|c|}
\hline \multirow[b]{2}{*}{ Space group } & \multirow[b]{2}{*}{$n \times c$-axis } & \multicolumn{2}{|c|}{$\omega_{1}($ at $A)$} & \multicolumn{2}{|c|}{$\omega_{2}($ at $A)$} & \multicolumn{2}{|c|}{$\omega_{3}($ at $A)$} & \multirow[b]{2}{*}{ Phonon calc'n } & \multirow[b]{2}{*}{ Funt'al } & \multirow[b]{2}{*}{$\Delta k$ value $\left(\AA^{-1}\right)$} \\
\hline & & $\left(\mathrm{cm}^{-1}\right)$ & $(\mathrm{meV})$ & $\left(\mathrm{cm}^{-1}\right)$ & $(\mathrm{meV})$ & $\left(\mathrm{cm}^{-1}\right)$ & $(\mathrm{meV})$ & & & \\
\hline$P 6 / \mathrm{mmm}$ & 1 & 236 & 29.3 & 331 & 41 & 398 & 49 & Linear & LDA & 0.01 \\
\hline$P \overline{6} c 2$ & 2 & 120 & 14.9 & 214 & 27 & 312 & 39 & Linear & LDA & 0.013 \\
\hline$P \overline{6} c 2$ & 2 & $117(12)$ & $15(2)$ & $210(5)$ & $26.0(6)$ & $303(13)$ & $38(2)$ & Finite D & $\mathrm{LDA}+\mathrm{GGA}$ & 0.01 \\
\hline$P \overline{3} m 1$ & 3 & 82 & 10 & 146 & 18 & 232 & 29 & Finite D & LDA & 0.01 \\
\hline$P \overline{3} m 1$ & 4 & $59(3)$ & $7.3(4)$ & $114(2)$ & $14.1(3)$ & $178(7)$ & $22(1)$ & Linear & $\mathrm{LDA}+\mathrm{GGA}$ & 0.01 \\
\hline
\end{tabular}

modes in order to achieve self-consistent models at the highest resolution possible for the supercell dimension.

Using different $k$-grid values and LDA and GGA approximations for the same superlattice model, a spread of phonon mode frequencies for the same mode is obtained. These values vary for specific modes at $\omega<400 \mathrm{~cm}^{-1}$, for example, frequency differences between $1 \mathrm{~cm}^{-1}$ and $3 \mathrm{~cm}^{-1}$ within the same approximation (LDA or GGA) for different $k$-grid values and differences between $\sim 5 \mathrm{~cm}^{-1}$ and $15 \mathrm{~cm}^{-1}$ for comparisons using LDA or GGA functionals for the same $k$-grid value. Readers are referred to earlier work on key parameter choices for DFT calculations that enable systematic evaluation of $\mathrm{meV}$ features pertinent to superconducting materials. ${ }^{27}$

For many converged calculations, the Density Functional Perturbation Theory (DFPT) approach was utilised. However, the $3 c$ calculation was unable to converge using this DFPT approach presumably due to inappropriate $k$-grid value $e^{27}$ or insufficient core and/or memory allocation for computation. Consequently, the finite displacement (FD) approach was utilised to achieve convergence for PD calculations for $2 c$ and $3 c$ (Table 2). The relevant PDs for $3 c$ and $4 c$ calculations are shown in Fig. S5 and S6 (ESI $\dagger$ ). An example of a converged PD calculated for superlattice period $2 c$, with Space Group symmetry $P \overline{6} c 2$ using a linear approximation for PD calculation is shown in Fig. $2 \mathrm{~b}$. Note the folding of reciprocal space in the $\Gamma A$ direction, which can be visualised by mirror reflecting the dispersion along $\Gamma A$ in Fig. 2a (i.e., for $P 6 / \mathrm{mmm}$ ), with a mirror plane along the dashed vertical line at $\Gamma A / 2$, which takes dispersion values on the Brillouin Zone (BZ) boundary at $A$ onto the $\Gamma$ point. While folding of reciprocal space predominantly takes place in the $\Gamma A$ direction, the bands along $K \Gamma$ and $\Gamma M$ are also influenced.

Table 2 lists DFT calculations obtained for the PD of $\mathrm{MgB}_{2}$ with symmetry conditions for three space groups: $P 6 / \mathrm{mmm}$, $P \overline{6} c 2$ and $P \overline{3} m 1$. The values for $\omega_{1}, \omega_{2}$ and $\omega_{3}$ are for the intersection of the acoustic phonons at the boundary $A$ along $\Gamma A$. Values from calculations that utilise the LDA and the GGA functional are averaged in Table 2. Standard deviations for the least significant value are given in parentheses. From Table 2, we note that for increasing value of $n$ (the superlattice along $c$ ) the value for $\omega_{1}$ decreases. In general, for $n=2$ and $n=4$, the lowest intersection of the acoustic phonon at the boundary $A$ (i.e., $\omega_{1}$ ) is approximately half of the lower order superlattice.

The accuracy of these DFT calculations, and thus, for frequency values, can also be evaluated from Table 2. For example, frequency values determined using an FD method for $n=2$ are within standard error of those obtained by a linear approximation at lower DFT resolution (i.e., where the value for $\Delta k$ is $\left.0.013 \mathrm{~A}^{-1}\right) .{ }^{27}$ We also note that the precision of calculations - as measured by the standard deviations for each frequency in Table 2 for the $n=4$ superlattice with $P \overline{3} m 1$ symmetry - suggests that this condition is optimum for the $\mathrm{MgB}_{2}$ structure. We show a partial PD for $\mathrm{MgB}_{2}$ with an $n=4 c$ superlattice in Fig. S6 (ESI $\dagger$ ).

\section{Discussion}

Frequencies relevant to the THz/Far IR regions are ideal for investigation of superlattice effects in the low wavenumber range. Wavenumber ranges for standard laboratory Fourier Transform IR (FTIR) and Raman spectroscopy typically extends to about $100 \mathrm{~cm}^{-1}$ or higher. Recent instrumentation advances can extend that limit to lower values for laboratory-based instruments with potential to measure below $50 \mathrm{~cm}^{-1} .^{29}$ Raman signals with low wavenumbers are common in artificial semiconductor nanostructures and can be explained as a result of superlattice periodicity. ${ }^{30-33}$ This periodicity folds reciprocal space and activates optic modes with phonon frequencies at fractional values of those for the original crystal periodicity. ${ }^{30-33}$

To place these experimental results into perspective, we examine possible mechanisms and evidence for superlattices in $\mathrm{MgB}_{2}$ and similar structures. We then relate the effect of superlattice geometries on subtle variations in electronic properties.

\section{Intrinsic superlattice periodicities - hybridised orbitals}

Previous discussion and graphic illustrations show that the electron density responsible for conduction in $\mathrm{MgB}_{2}$ is largely concentrated along B-B covalent bonds. ${ }^{9,27,28}$ These electronic charges populate $\sigma$-bands which correspond to the inverted, approximately parabolic bands ${ }^{30,31}$ that are degenerate at $\Gamma$.

Consideration of more than one unit cell as shown schematically for the $a-b$ plane of $\mathrm{MgB}_{2}$ in Fig. 3 (emphasizing hybridisation between boron atoms) provides a convenient means to visualise the relationship of bonding and antibonding orbitals in real space. DFT calculations ${ }^{31}$ of $\mathrm{MgB}_{2}$ band structures identify these $\sigma$-bands at $\Gamma$ as shown in Fig. S7 (ESI $\dagger$ ), and through consideration of bonding and anti-bonding orbitals, translates to the hybridized orbital configurations shown in Fig. $3 .^{31}$

This bonding and anti-bonding orbital character provides a real space physical interpretation for the origin of approximate parameters that define the inverted parabolic construct at $\Gamma$ in 


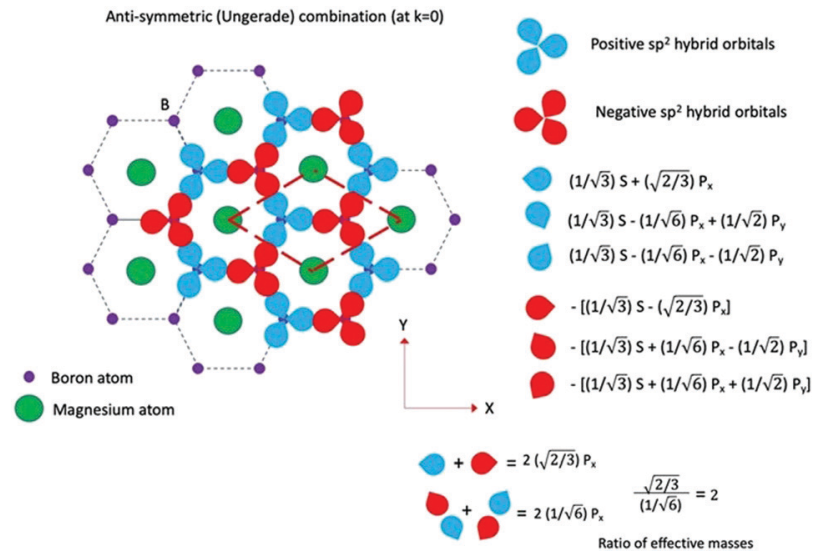

Fig. 3 Schematic diagram of anti-bonding $\mathrm{sp}^{2}$ hybridisation within the boron plane(s) of $\mathrm{MgB}_{2}$

band structure calculations for $\mathrm{MgB}_{2} \cdot{ }^{31,32}$ We have developed these parameters to determine differences between heavy and light effective masses for $\mathrm{MgB}_{2}$ around $\Gamma$ and for associated bands. ${ }^{32}$ This interpretation of band structures is well understood for semiconductors with analogous, degenerate $\sigma$-bands $s^{33-36}$ and similar principles are utilised in this study. For completeness, we detail an equivalent schematic for $\mathrm{sp}^{2}$ bonding hybridisation within the boron plane(s) of $\mathrm{MgB}_{2}$ in Fig. S8 (ESI $\dagger$ ).

The anti-bonding character of $\sigma$-bands at $\Gamma$ is also supported by DFT calculations which show that the energy for these bands is higher at $\Gamma$, compared to their energy as the bands circumscribe bonding character at the boundary zone ${ }^{37}$ (e.g., at $M$ along $\Gamma M$ or at $K$ along $\Gamma K$ ). At these points on the boundary zones, a change in phase is also expected. In contrast, along the $\Gamma A$ direction, the $\sigma$-band has lower energy at $\Gamma$ compared to the energy at $A$, so an opposite condition along the $c^{*}$ direction is expected. In this case, a bonding orbital with $\mathrm{p}_{z}$ character at $\Gamma$ and with antibonding character at $A$ is evident (Fig. S7, ESI $\dagger$ ).

One possible interpretation of real space bonding character, consistent with anti-bonding $\mathrm{sp}^{2}$ hybridisation in the boron plane, and with bonding character in the $c$-direction (at $\Gamma$ ) is schematically illustrated in Fig. 4. In this interpretation, we posit that by considering the bonding-antibonding character of orbital hybridisation in the boron plane and in the $c$-direction, phase changes resulting in a halving of the $c^{*}$-direction (or doubling of the $c$ axis lattice parameter) is a minimal requirement to define an appropriate structural periodicity.

With electrons at the Fermi energy (i.e., the conduction electrons) localised along $\mathrm{B}-\mathrm{B}$ bonds, ${ }^{28}$ periodicities in real space that are modulated by the orbital character of boron atoms can be identified. These periodicities appear to be correlated to wavelengths associated with Bloch waves. For instance, from a knowledge of the Fermi wavevector, determined from EBS intersections with the Fermi energy and/or their relative distances with respect to the BZ boundary in Fermi surfaces (FS), simple relationships are apparent in reciprocal space. These relationships are demonstrated schematically in Fig. S9 (ESI $\dagger$ ). As is readily demonstrated in textbook examples, ${ }^{38,39}$ these fractional reciprocal space dimensions can be converted to in-plane superlattice modulations and to distances in real space. ${ }^{38,40-42}$

The average radius of the tubular FS for $k_{z}=0$ is $\sim 1 / 6$ of the distance from the $\Gamma$ centre point to the $M$ point of the BZ
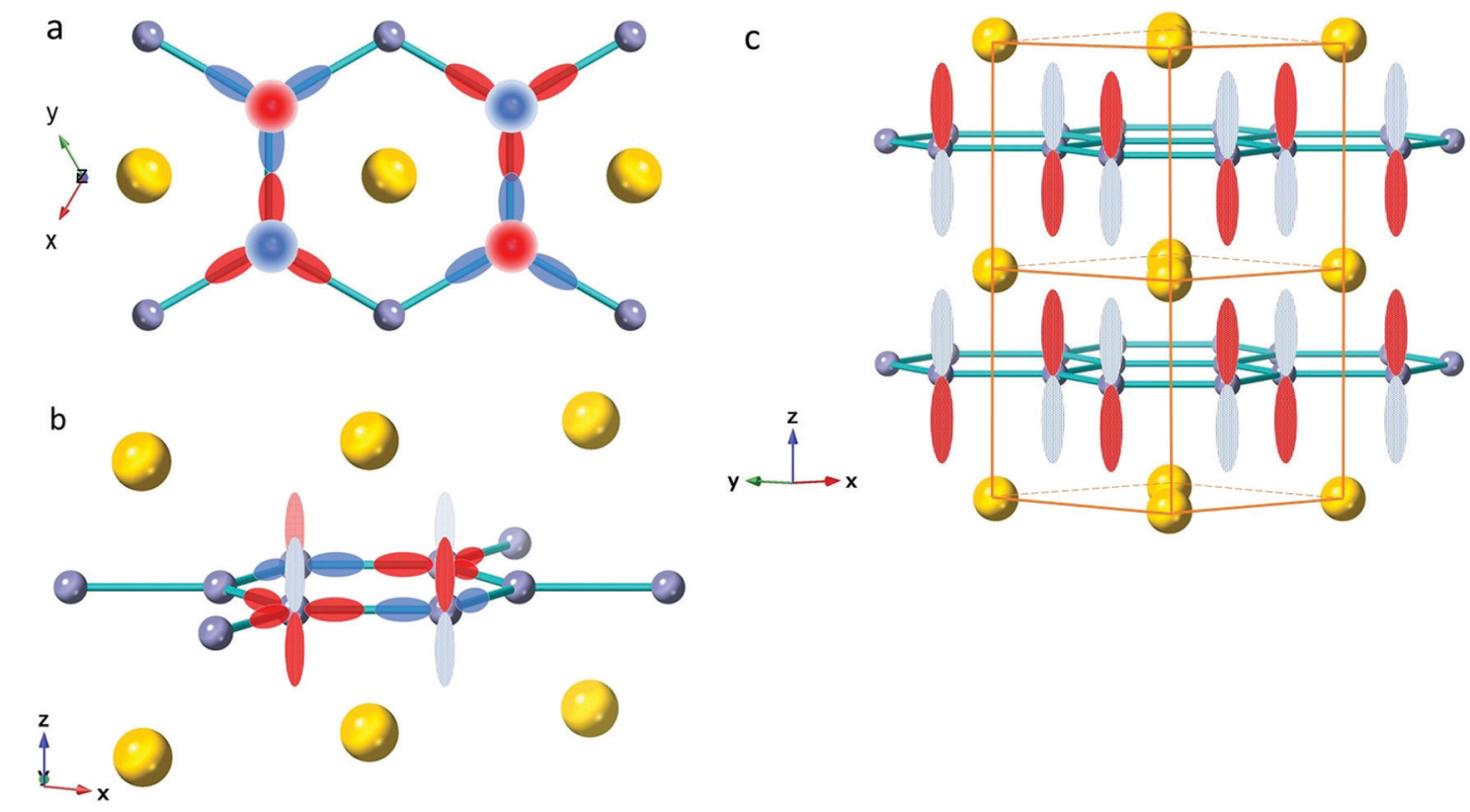

Fig. 4 Schematic diagram of $p_{z}$ hybridised orbitals associated with $\sigma$-bands (at $k=0$ ) (a) projection down [001] showing bonding (blue) and antibonding (red) orbitals above/below boron sites; (b) same construct as in (a) with perspective view at a $7^{\circ}$ tilt angle to [010] in the $x-z$ plane showing orientation of $\mathrm{p}_{z}$ hybrid orbitals ( $\mathrm{Mg}-\mathrm{B}$ bonds and some $\mathrm{Mg}$ atoms omitted for clarity) and (c) perspective view at a $5^{\circ}$ tilt to [110] showing adjacent boron planes of $\mathrm{MgB}_{2}$. Hybrid $\mathrm{sp}^{2}$ orbitals in the B-B plane are omitted for clarity (assumes no involvement of $\mathrm{Mg}$ orbitals in this bonding schema). A right-handed set of all positive or negative sign is assumed for $\mathrm{sp}^{2}$ bonds and $\mathrm{p}_{z}$ at each $\mathrm{B}$ atom. 
boundary. This radius translates to a wavelength six times the inverse of the reciprocal space distance to the boundary. At reciprocal point $A$ (i.e., $k_{z}=\pi / c$ ), the FS shows an increased radius to $\sim 1 / 4$ of the $\Gamma M$ distance. Careful inspection of the parallel FSs (Fig. S9, ESI $\dagger$ ) shows that the radius of the outer (heavy effective mass) FS at $k_{z}=0$ is approximately identical to the radius of the inner (light effective mass) FS at $k_{z}=\pi / c$.

This observation is a key to concepts of electron resonance, whereby conservation of energy and momentum (or wavevector) is inherently guaranteed by a constant value for the Fermi energy and the total momentum of combined Fermi wavevectors. For example, for electrons with states at half the distance to the $A$ point $\left(k_{z}=\pi / 2 c\right)$, the $a^{*}$ (or $x$-vector) component of the Fermi wavevector is given by,

$$
k_{F x}=[(1 / 4+1 / 6) / 2] \Gamma M=5 / 24 \Gamma M .
$$

Two electrons at this state may interact to produce one electron at $k_{z}=0$ with $k_{F x}=1 / 6 \Gamma M$ and another at $k_{z}=\pi / c$ with $k_{F x}=1 / 4 \Gamma M$. Thus, a mechanism to transfer momentum between a direction fully contained in the $a^{*}-b^{*}$ plane will include energy components in the $c^{*}$ direction(s). In the $\Gamma K$ direction, different multiplicities exist at $k_{z}=0$ and $k_{z}=\pi / c$ as shown in ESI, $\dagger$ Fig. S9. In this case, further interplay of electrons between the $\Gamma M$ and $\Gamma K$ directions is also probable.

We note that integer multiples of the $c$-axis lattice parameter can be matched to periodicities identified in the hybridisation schematic and in the analysis of average FS radii relative to the BZ boundary. For example, $3 c /\left(3^{1 / 2} a\right)=3.5 \AA \times 3 /\left(3.04 \AA \times 3^{1 / 2}\right)=$ $10.5 / 5.265=1.994 \sim 2$. Such proportionality between $c$-axis dimensions and in-plane periodicities favours a match between in-plane and perpendicular acoustic vibrations and velocities as discussed in the following section.

\section{Acoustic phonons and lattice dimensions}

Above absolute zero, the Bose-Einstein statistical distribution applies to both longitudinal and transverse acoustic phonons in all possible three-dimensional (3D) directions. For the layered hexagonal structure of $\mathrm{MgB}_{2}$, a longitudinal acoustic movement in the $a^{*}-b^{*}$ plane is oriented parallel to transverse acoustic movements along the $c^{*}$ direction and vice versa.

Longitudinal vibrations are compression waves and transverse vibrations are shear waves resulting in stronger restoring forces for the former compared to the latter vibrations. ${ }^{43}$ Appropriate consideration of in-phase (i.e., same sense) movement of the entire unit cell is required for correct analysis of acoustic modes. In contrast, optical modes, or acoustic modes at the BZ boundary, demonstrate an anti-sense (or out of phase) movement. In these latter cases, atoms in the basis set move relative to each other out of phase. ${ }^{43}$ DFT calculations show that $\mathrm{Mg}$ is the major contributor to the PD partial density of states in the acoustic region. ${ }^{10}$ Thus, movement of $\mathrm{Mg}$ atoms will influence the nature of acoustic phonon interactions and, as we will show, are an additional low energy (meV) feature of $\mathrm{MgB}_{2}$ superconducting properties.
Inspection of the PD for $\mathrm{MgB}_{2}$ with $P 6 / \mathrm{mmm}$ symmetry as shown in Fig. 2a, shows that the acoustic transverse and longitudinal frequencies for $k_{z}=\pi / 2 c$ (or $\Gamma A / 2$ ) display harmonic behaviour up to the second lowest optical mode (i.e., $\sim 415 \mathrm{~cm}^{-1}$ ). Furthermore, the two intersecting acoustic phonon frequencies at $\Gamma A / 2$ show similar values to two other, inplane high symmetry directions at $\Gamma M$ and $\Gamma K$. Remarkably, these matching values occur at $\sim 1 / 3 \Gamma M$ and $1 / 4 \Gamma K$, respectively. In practice, these values are in equivalent reciprocal space positions along the linear sections (i.e., parallel to the Fermi level) of the PD anomaly associated with the $\mathrm{E}_{2 \mathrm{~g}}$ mode ${ }^{10-12}$ as shown by the dotted vertical lines in Fig. 2 a.

In Fig. 2a, for a given frequency $\omega,\left(\right.$ e.g.,$\sim 100 \mathrm{~cm}^{-1}$ to $\sim 110 \mathrm{~cm}^{-1}$, we identify that the $k$-vector at which the longitudinal acoustic branch along $\Gamma A$ shows this frequency value is $\sim \Gamma A / 4$. Accordingly, intersection of this same frequency with the transverse acoustic branch is at $\sim \Gamma A / 2$. This relationship confirms that wavelengths for the respective acoustic mode wavevectors show inverse proportionality with a ratio $\sim 2$. That is, the longitudinal acoustic velocity is approximately twice the value of the transverse acoustic velocity at the same frequency. This proportion results from the balance of restoring forces along longitudinal and transverse directions. Similar conclusions can be obtained for the $\Gamma M$ and $\Gamma K$ directions shown in Fig. 2a.

Our earlier work inferred the presence of partial harmonicity for acoustic PDs. ${ }^{19,44}$ With this in mind, we note that direct and reciprocal space proportional dimensions favour the occurrence of standing waves in crystalline structures. Such standing waves generate non-dispersive waves in regions where the dispersion curve, $\omega$ vs. $k$, has approximately linear dependence. ${ }^{10,45}$ In this manner, coincident lattice points for electronic and acoustic waves may serve as nodal points, enabling a degree of tuning and synchronicity between electron and phonon waves that move and interact across the material. However, the observation of superlattice frequencies, which may be difficult to detect but are nevertheless present, may be an indication of minor anharmonicity in $\mathrm{MgB}_{2}$. In such a case, multiples of a single harmonic are required to better approximate anharmonic behaviour. ${ }^{45}$ Anharmonicity has been reported in several publications ${ }^{46-48}$ as strongly linked to superconductivity in $\mathrm{MgB}_{2}$; this connection to superlattices is discussed below.

\section{Superlattice signatures}

A mechanistic interpretation of DFT calculations for superconductivity can also be gained by further analysis of electron density distributions and Fermi surface topologies. ${ }^{28,49,50}$ In particular, these features provide detail about the electrons that participate in conduction processes. ${ }^{38,40-42}$ The deformation potential approach has clearly shown that the conduction electrons in $\mathrm{MgB}_{2}$ largely move in the boron planes, along $\mathrm{B}-\mathrm{B}$ bonds in response to $\mathrm{B}$ atom movements along $\mathrm{E}_{2 \mathrm{~g}}$ optical phonon mode displacement patterns. ${ }^{9,28}$ For acoustic displacements along the boron in-plane directions, stretching of the B-B bonds is also expected to result in deformation potentials. Thus, we may observe a corresponding modulated electron 
density distribution, or charge density wave, as inferred in our earlier work. $^{28}$

A net electric field pointing along the $c$-direction is generated because the electron density response to acoustic (and optic vibration) deformation is largely contained within the $\mathrm{B}-\mathrm{B}$ plane. ${ }^{28}$ An electric field effect is also generated because electron density modulations retain connection to the periodicity and displacement symmetry of the in-plane longitudinal acoustic wave. ${ }^{51}$ This electric field imparts a driving force onto the charged $\mathrm{Mg}$ layers which donate their valence electrons to the B-B plane. ${ }^{8,9}$ This effect establishes a strong coupling between acoustic movements in the B-B plane and the perpendicular plane (i.e., the $c$ - or $c^{*}$-direction). This coupling is largely reflected in the coincidence of velocities along two different directions as noted above. This coincidence of velocities may also guarantee a high degree of coherence and synchronicity that would otherwise not be possible without the influence of acoustic phonon modes.

The fact that we observe absorption of THz/Far IR radiation in spectra of $\mathrm{MgB}_{2}$ at frequencies that include $100-110 \mathrm{~cm}^{-1}$ but also at substantial fractions of these values is essential to our understanding of superconductivity. For instance, these observations indicate that all these low frequencies contribute to the available optic modes of $\mathrm{MgB}_{2}$ that absorb electromagnetic radiation on approaching $T_{\mathrm{c}}$ and at values below $T_{\mathrm{c}}$. This feature also confirms that superlattices are present in the material, as suggested from our earlier Raman experiments. ${ }^{19,20}$

Furthermore, these acoustic modes must have a strong connection to superconductivity given that the spectral density largely evolves after crossing $T_{\mathrm{c}}$. These superlattice signatures are indicative of an overall structural transition with significant electronic involvement which we suggest is connected to the anharmonicity of $\mathrm{MgB}_{2} \cdot{ }^{45-48}$ Small gaps may also be expected in the electronic band structures for superlattices at the new superlattice BZ boundaries. ${ }^{17,19,20,22}$ We also suggest that previous interpretations of frequencies matching superconducting gap energies in Raman investigations may have mistakenly attributed such observations to pair breaking mechanisms. Pair breaking mechanism(s) can not readily explain observations at fractional values of the presumed pair-breaking (gap) frequencies.

Superlattice formation has been experimentally confirmed in Al-substituted $\mathrm{MgB}_{2}$ by spectroscopy ${ }^{10}$ and by electron microscopy. ${ }^{52,53}$ Considering the $\mathrm{AlB}_{2}$-rich end of the substitutional range for this structure type, amplification of the superconducting $T_{\mathrm{c}}$ has been discussed by DiCastro et al. ${ }^{54,55}$ in terms of a combined effect of tuning the Fermi level and the tensile micro-strain. The importance of a Feshbach shape resonance ${ }^{56-58}$ and proximity of the chemical potential (or FS) to a topological Lifshitz transition has been extensively discussed by Bianconi ${ }^{50}$ and constitutes a topic of ongoing interest at SuperStripes conferences. We also note sub-THz reflectivity ratios obtained for CaAlSi, a superconductor $\left(T_{\mathrm{c}}=6.7 \mathrm{~K}\right)$ isostructural with $\mathrm{MgB}_{2}$, described by Lupi. ${ }^{17}$ With radiation polarized along the $c$ axis direction, the reflectivity ratio shows a series of peaks between $10 \mathrm{~cm}^{-1}$ and $40 \mathrm{~cm}^{-1}$, particularly for data at $3.5 \mathrm{~K}$ and $4.5 \mathrm{~K} .{ }^{17}$ Such modularity of signal(s) below the $T_{\mathrm{c}}$ value for CaAlSi may be indirect evidence for the presence of a lower symmetry superlattice at these temperatures.

The investigation of Al-substituted $\mathrm{MgB}_{2}$ using high resolution neutron diffraction with pair distribution functions by Campi et al., ${ }^{22}$ is also a source of relevant structural information. For example, comparison of the $c$-axis value for $\left(\mathrm{Mg}_{1-x} \mathrm{Al}_{x}\right) \mathrm{B}_{2}$ at superlattice multiples of $n(n=2,3,4)$ showed that when $x=0.5$, the superlattice $c$-axis is an integer multiple of the fundamental unit cell. However, for $x=0.0$ (i.e., no Al substitution) and for intermediate values of $x$, a four times $c$ axis superlattice for $\mathrm{MgB}_{2}$ diverged from an integer multiple beyond the experimental errors of these high resolution structural determinations. ${ }^{22}$ These data by Campi et al. ${ }^{22}$ suggest that $\mathrm{MgB}_{2}$ may exist as a supercell, wherein particular internal distances are shifted slightly from $P 6 / \mathrm{mmm}$ positions.

More recently, Wu et al. ${ }^{59}$ used transmitted X-ray diffraction measurements with synchrotron radiation to explore electron and hole densities in $\mathrm{MgB}_{2}$ for temperatures $6 \mathrm{~K}<T<100 \mathrm{~K}$. Wu $e t ~ a l .{ }^{59}$ used a difference electron Fourier method and determined that electron and hole densities develop an asymmetric distribution with respect to the $\mathrm{B}$ atomic plane. This asymmetry is accompanied by differentiation of the distances between the B-B plane and adjacent $\mathrm{Mg}$ layers, as the temperature approaches and crosses the superconducting transition temperature. We interpret these observations to suggest a change in symmetry of the $\mathrm{MgB}_{2}$ structure occurs as temperature is reduced to $T_{\mathrm{c}}$ and to values below $T_{\mathrm{c}}$. This change is consistent with a lower, or reduced, symmetry indicative of a superlattice structure. ${ }^{19}$

Finally, circumstantial evidence for superlattice formation may be invoked from the enhanced signal strength and distinctive features of the spectra for C-doped $\mathrm{Mg}^{11} \mathrm{~B}_{2}$ compared with $\mathrm{MgB}_{2}$. Earlier work $^{23,24}$ established that carbon in $\mathrm{Mg}\left(\mathrm{B}_{1-x} \mathrm{C}_{x}\right)_{2}$ for $0<x<0.3$ substitutes onto boron sites in the $a-b$ plane. In this study, for which $x=0.10$, an ordered distribution of carbon in the $a-b$ plane will disturb the $P 6 / \mathrm{mmm}$ symmetry compared to $\mathrm{MgB}_{2}$ without carbon.

The symmetry of the hexagonal plane is compared in Fig. 5 in which we show ordered arrangements of 30 atoms with nominal P6/ mmm symmetry. In Fig. 5a, we show a normal distribution of boron atoms including the natural abundance of both isotopes. In Fig. 5b, we show the presence of $10 \% \mathrm{C}$ on boron sites in an ordered configuration that requires one $\mathrm{C}$ to be alternately on either side of the (110) plane as displayed by the grey coloured atoms (i.e., a mirror plane is removed). This requirement clearly violates $P 6 / \mathrm{mmm}$ symmetry and, we suggest, may be a superlattice configuration with P6 $\mathrm{mm}$ symmetry as noted in an earlier publication. ${ }^{19}$ Changes to lower symmetry conditions - induced by a different atom - may accentuate $\mathrm{THz}$ signals for $\mathrm{C}$-doped $\mathrm{Mg}^{11} \mathrm{~B}_{2}$ compared to those obtained for $\mathrm{MgB}_{2}$. These variations in $\mathrm{THz}$ spectral signal at low wavenumber for substituted compositions of the $\mathrm{AlB}_{2}$ type structure warrants further detailed understanding.

\section{Analogous band structures}

The connection between superlattices and superconductivity of $\mathrm{MgB}_{2}$ and C-doped $\mathrm{MgB}_{2}$ is clearly evident by these $\mathrm{THz} / \mathrm{Far}$ IR 
a

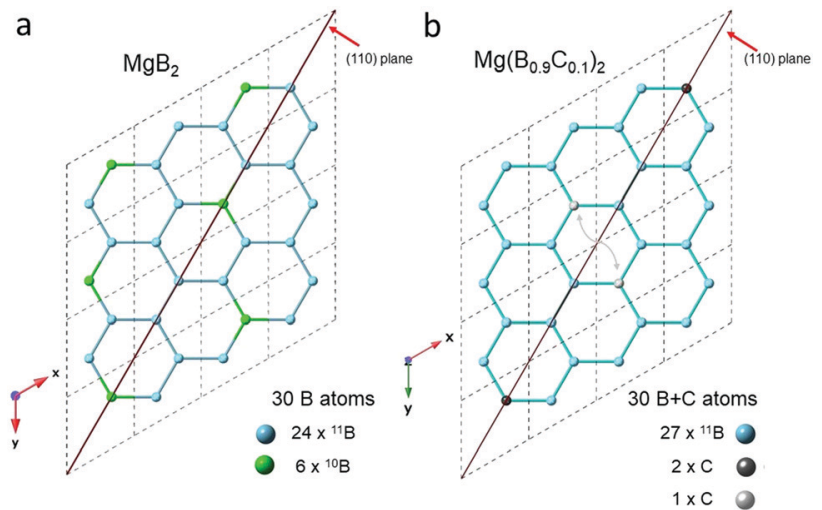

Fig. 5 Schematic of $\mathrm{B}-\mathrm{B}$ bonding within the $a-b$ plane for 16 unit cells of (a) $\mathrm{MgB}_{2}$ and (b) $M g\left(B_{0.9} C_{0.1}\right)_{2}$. This schematic shows an ordered distribution of 30 atoms which retains nominal $P 6 / \mathrm{mmm}$ symmetry in (a), and a lower symmetry for (b) due to $C$ atom substitution for $B$ in the $a-b$ plane. For an ordered structure in the $a-b$ plane, one $C$ atom must be alternately sited on either side of the (110) plane (denoted by the grey atoms in (b)) which removes a mirror plane from $P 6 / \mathrm{mmm}$ symmetry.

absorption measurements with change in temperature to, and below, $T_{\mathrm{c}}$. However, there is a similarity of topology between key $\mathrm{MgB}_{2} \sigma$-bands and the bands calculated for artificial semiconductor superlattices. In particular, an analogy to semiconductor superlattices perpendicular to the growth direction is encapsulated in corresponding Tight Binding (TB) equations. For example, in the vicinity of the Fermi energy, the approximately inverted parabolic $\sigma$-bands can be mathematically expressed using TB equations for layered materials ${ }^{31,60-63}$ as

$$
E_{n}\left(k_{x}, k_{y}, k_{z}\right)=E_{0}-2 t_{\perp} \cos \left(c k_{z}\right)-\hbar^{2}\left(k_{x}^{2}+k_{y}^{2}\right) / 2 m_{n}
$$

where the index $n$ labels the heavy/light hole bands, $m_{n}$ are the respective in-plane effective masses and $t_{\perp}$ is the transverse transfer matrix element from one layer to another which characterizes the intensity of electron tunnelling between layers.

For $\mathrm{MgB}_{2}$, the effective masses for the heavy and light holes are typically around $m_{\text {eff }}^{H}=0.53-0.59, m_{\text {eff }}^{L}=0.20-0.28, E_{0}=0.58-0.6$ and $t_{\perp}=0.092-0.094 \mathrm{eV} .^{9,64,65}$ Apart from the specific coefficients, the TB equation is identical to the equation used to describe artificial semiconductor superlattices, where $c$ is perpendicular to the superlattice interface (i.e., parallel to the growth direction). ${ }^{66,67}$

According to these TB equations, electrons move nearly freely in the $a-b$ plane and tunnel across potential barriers in the $c$-direction. ${ }^{66,67}$ Since the effective mass in the $c$-direction is

$$
m_{\mathrm{eff}}=\hbar^{2}\left(\partial^{2} E / \partial k^{2}\right)^{-1},
$$

we have,

$$
m_{\mathrm{eff}}=\hbar^{2}\left(2 c^{2} t_{\perp} \cos \left(c k_{z}\right)\right)
$$

Hence, $m_{\text {eff }}$ is undefined (or infinite) when $c k_{z}=(2 n-1) \pi /$ $2(n=1,2,3, \ldots, \infty) .{ }^{68}$ From the gradient of the FS,

$$
E_{n}\left(k_{x}, k_{y}, k_{z}\right)=\left(-\frac{\hbar^{2} k}{m_{n}},-\frac{\hbar^{2} k_{y}}{m_{n}}, 2 c t_{\perp} \sin \left(c k_{z}\right)\right)
$$

it can be clearly seen that

$$
c k_{z}=(2 n-1) \pi / 2(n=1,2,3, \ldots, \infty)
$$

are the points of maximum $k_{z}$ gradient and that

$$
c k_{z}=n \pi(n=1,2,3, \ldots, \infty)
$$

are points where the gradient has zero $k_{z}$ component. Therefore, the mid position to the BZ boundary in the $c^{*}$-direction displays a singularity. At this singularity, the FS undergoes an inflection of curvature which may be significant for interpretation of the dynamics of electron movement in both semiconductor superlattices and superconductors.

Under these conditions, it is worth noting that the mid position to the $\mathrm{BZ}$ boundary at $\mathrm{A}$ for $\mathrm{MgB}_{2}$, with $k_{z}=\pi / 2 c$, is again of significance, but from a different perspective given our analogy to semiconductor superlattices. For example, a well known feature of artificial semiconductor superlattices is that folding of reciprocal spaces for individual semiconductor components is displayed when the combined nanoscale thicknesses of alternating semiconductor materials becomes the new superlattice unit cell dimension. ${ }^{35,66,67,69}$ Such folding influences many semiconductor properties and is manifest as lower vibration frequencies in Raman and IR spectra. ${ }^{32,35,70,71}$ This understanding of the $\mathrm{BZ}$ condition along $\Gamma-A$ is consistent with our previous published Raman data on $\mathrm{MgB}_{2}{ }^{19}$ and $\mathrm{YB}_{6},{ }^{20}$ as well as the current synchrotron THz/Far IR observations. Not unreasonably, we also expect that other quasiparticles, or excitations in the meV energy range, observed and extensively investigated for artificial semiconductor superlattices, may influence our understanding of superconductivity in $\mathrm{MgB}_{2}$ and many other materials.

\section{Conclusions}

$\mathrm{THz} /$ Far IR synchrotron transmission experiments as a function of temperature have been conducted on diluted, pelletised $\mathrm{MgB}_{2}$ and C-doped $\mathrm{Mg}^{11} \mathrm{~B}_{2}$. Calibrated absorption spectra at low wavenumber display a clear correlation with superconductivity, as sample temperature reduces to near, and then below, $T_{\mathrm{c}}$. The intensity of the absorption peaks and the overall spectral weight of the THz/Far IR signals increase with lower sample temperature. Strong absorption occurs at wavenumber values that correspond to superconducting gaps for C-doped $\mathrm{Mg}^{11} \mathrm{~B}_{2}$ and $\mathrm{MgB}_{2}$. Absorption peaks are also observed at substantial fractions of these values, indicating that superlattice frequencies contribute to the PD for these superconductors by introducing new optic modes. DFT calculations using superlattices along the $c$-axis direction show folded mode frequencies in PDs that match observed experimental absorption values.

Analyses of bonding characteristics, Fermi vectors from electronic band structures and Fermi surfaces, PD relations and geometrical parameters, enable identification of integer proportionalities in real and reciprocal spaces that are consistent with subtle shifts in real space symmetry. For example, these analyses indicate that symmetry reductions from $P 6 / \mathrm{mmm}$, 
largely electronic in character though coupled to phonon frequencies, occur in $\mathrm{MgB}_{2}$ and $\mathrm{C}$-doped $\mathrm{Mg}^{11} \mathrm{~B}_{2}$ when cooled near to and below $T_{\mathrm{c}}$. Such symmetry reductions have strong implications for interpretation of superconducting mechanisms. We reflect on the similarities in our interpretations with physical property manifestations from artificial semiconductor superlattices. The presence of low wavenumber peaks clearly coincident with independently determined superconductor energy gaps for $\mathrm{MgB}_{2}$ and C-doped $\mathrm{Mg}^{11} \mathrm{~B}_{2}$ suggest that superlattices may be exploited to design new superconductors to achieve desired values of $T_{\mathrm{c}}$. Analogies with artificial semiconductor superlattices may provide further guidance and inspiration for superconductor materials development.

\section{Author contributions}

JAA: conceptualisation, formal analysis, methodology, investigation, supervision, funding acquisition, writing - original, review and editing; BG: formal analysis, investigation, validation and visualization; MS: formal analysis, investigation, resources, validation; DA: investigation, methodology and supervision; IDRM: conceptualisation, formal analysis, funding acquisition, validation, visualization, writing - review and editing.

\section{Conflicts of interest}

There are no conflicts to declare.

\section{Acknowledgements}

The authors are thankful to the Australian Synchrotron, ANSTO for awarded merit beamtime. We also acknowledge the Central Analytical Research Facility (CARF) and the e-Research Office, both at QUT, for access to laboratory facilities and to high performance computing and assistance, respectively.

\section{References}

1 P. B. Allen, in Dynamical Properties of Solids, ed. G. K. Horton and A. A. Maradudin, North Holland Publishing Company, 1980, vol. 3, ch. 2 .

2 J. Bardeen, L. N. Cooper and J. R. Schrieffer, Phys. Rev., 1957, 108, 1175.

3 M. Tinkham, Introduction to Superconductivity, McGraw-Hill, New York USA, 1996.

4 E. Maxwell, Phys. Rev., 1950, 78, 477.

5 C. Reynolds, B. Serin and L. Nesbitt, Phys. Rev., 1951, 84, 691.

6 A. Bill, V. Kresin and S. Wolf, Pair Correlations in ManyFermion Systems, Springer, 1998, p. 25.

7 R. F. Wood, B. E. Sernelius and A. L. Chernyshev, Phys. Rev. B: Condens. Matter Mater. Phys., 2002, 66, 1.

8 J. Kortus, I. I. Mazin and K. D. Belashchenko, et al., Phys. Rev. Lett., 2001, 86, 4656.

9 J. M. An and W. E. Pickett, Phys. Rev. Lett., 2001, 86, 4366.
10 J. A. Alarco, P. C. Talbot and I. D. R. Mackinnon, Phys. Chem. Chem. Phys., 2015, 17, 25090.

11 I. D. R. Mackinnon, P. C. Talbot and J. A. Alarco, Comput. Mater. Sci., 2017, 130, 191.

12 J. A. Alarco, P. C. Talbot and I. D. R. Mackinnon, Phys. C, 2017, 536, 11.

13 W. Ghann and J. Uddin, Terahertz (THz) Spectroscopy: A Cutting-Edge Technology, InTechOpen, 2016.

14 G. L. Carr, M. C. Martin and W. R. McKinney, et al., Nature, 2002, 420, 153.

15 O. Khatib, H. A. Bechtel and M. C. Martin, et al., ACS Photonics, 2018, 5, 2773.

16 Y.-L. Mathis, B. Gasharova and D. Moss, J. Bilogical Phys., 2003, 29, 313.

17 S. Lupi, in Advances in Condensed Matter Physics, ed. V. V. Moshchalkov, Hindawai Publishing Corporation, 2011, ch. Article ID 816906, p. 9.

18 P. Calvani, S. Lupi and M. Ortolani, et al., Infrared Phys. Technol., 2008, 51, 429.

19 J. A. Alarco, A. Chou and P. C. Talbot, et al., Phys. Chem. Chem. Phys., 2014, 16, 24443.

20 J. A. Alarco, M. Shahbazi and P. C. Talbot, et al., J. Raman Spectrosc., 2018, 49, 1985.

21 S. J. Clark, M. D. Segall and C. J. Pickard, et al., Z. Kristallogr., 2005, 220, 567.

22 G. Campi, A. Ricci and A. Bianconi, J. Supercond. Novel Magn., 2012, 25, 1319.

23 J. Karpinski, N. D. Zhigadlo and S. Katrych, et al., Phys. C, 2007, 456, 3.

24 J. Karpinski, N. D. Zhigadlo and S. Katrych, et al., Phys. Rev. B: Condens. Matter Mater. Phys., 2008, 77(214507), 1-11.

25 J. C. Grivel and M. Burdusel, Phys. C, 2016, 528, 65.

26 J. A. Alarco and I. D. R. Mackinnon, in Phonons in Low Dimensional Structures, ed. V. N. Stavrou, InTech Open, London UK, 2018, ch. 5, p. 75.

27 I. D. R. Mackinnon, A. Almutairi and J. A. Alarco, in Real Perspectives of Fourier Transforms and Current Developments in Superconductivity, ed. J. M. V. Arcos, IntechOpen Ltd., London UK, 2021, ch. 10, p. 1.

28 J. A. Alarco, P. C. Talbot and I. D. R. Mackinnon, Mod. Numer. Sim. Mater. Sci, 2018, 8, 21.

29 H. J. Choi, D. Roundy and H. Sun, et al., Nature, 2002, 418, 758.

30 D. S. Urch, Orbitals and Symmetry, Penguin Books Ltd, Harmondsworth, Middlesex, England, 1970.

31 A. Almutairi, Electronic Band Structure Equations and Fermi Surface Evolution from $2 D$ Materials to $3 D$ Layered Superconducting Compounds, PhD thesis, Queensland University of Technology, 2019.

32 M. Dresselhaus, G. Dresselhaus and S. B. Cronin, et al., Solid State Properties - From Bulk to Nano, Springer Nature, Berlin, Germany, 2018.

33 J. C. Phillips, Bonds and Bands in Semiconductors, Academic Press, 1973.

34 M. I. Dyakonov, Spin Physics in Semiconductors, Springer, 2nd edn, 2017. 
35 in Fundamentals of Semiconductors - Physics and Materials Properties, ed. P. Y. Yu and M. Cardona, Springer, 2010.

36 K. W. Böer and U. W. Pohl, Semiconductor Physics, Springer, 2018.

37 J. Stohr and H. C. Siegmann, Magnetism - From Fundamentals to Nanoscale Dynamics, Springer, 2006.

38 S. L. Altmann, Band Theory of Solids: An Introduction from the Point of View of Symmetry, Clarendon Press, Oxford, 2002.

39 H. Jones, The Theory of Brillouin Zones and Electronic States on Crystals, North-Holland Publishing Company, Amsterdam, 1960.

40 N. W. Ashcroft and N. D. Mermin, Solid State Physics, Saunders, Philadelphia, 1976.

41 C. Kittel, Introduction to solid state physics, Wiley, 2005.

42 J. M. Ziman, Principles of the Theory of Solids, Cambridge University Press Second edn, 1972.

43 J. M. Ziman, Electrons and Phonons: The Theory of Transport Phenomena in Solids, Oxford University Press, 1960.

44 J. A. Alarco, P. C. Talbot and I. D. R. Mackinnon, Phys. Chem. Chem. Phys., 2014, 16, 25386.

45 I. G. Main, Vibrations and Waves in Physics, Cambridge University Press, Cambridge, 1979.

46 T. Yildirim, O. Gülseren and J. W. Lynn, et al., Phys. Rev. Lett., 2001, 87, 037001.

47 M. d'Astuto, M. Calandra and S. Reich, et al., Phys. Rev. B: Condens. Matter Mater. Phys., 2007, 75, 1.

48 A. Y. Liu, I. I. Mazin and J. Kortus, Phys. Rev. Lett., 2001, 87, 087005.

49 J. A. Alarco, A. Almutairi and I. D. R. Mackinnon, J Supercond Nov Magn, 2020, 33, 2287.

50 A. Bianconi, J. Supercond. Novel Magn., 2018, 31, 603.

51 G. Grüner, Density waves in solids, 1994.

52 H. W. Zandbergen, M. Y. Wu and H. Jiang, et al., Phys. C, 2002, 366, 221.

53 J. Y. Xiang, D. N. Zheng and J. Q. Li, et al., Phys. Rev. B: Condens. Matter Mater. Phys., 2002, 65, 214536.
54 D. Di Castro, S. Agrestini and G. Campi, et al., Europhys. Lett., 2002, 58, 278.

55 A. Bianconi, S. Agrestini and D. Di Castro, et al., Phys. Rev. B: Condens. Matter Mater. Phys., 2002, 65, 174515.

56 A. Bianconi, D. Di Castro and S. Agrestini, et al., J. Phys.: Condens. Matter, 2001, 13, 7383.

57 A. Bianconi, J. Supercond.: Incorporating Novel Magnetism, 2005, 18, 626.

58 A. Bianconi, S. Agrestini and A. Bussmann-Holder, J. Supercond., 2004, 17, 205.

59 S. Y. Wu, P.-H. Shih and J.-Y. Ji, et al., Supercond. Sci. Technol., 2016, 29, 045001.

60 I. N. Askerzade and B. Tanatar, Phys. C, 2003, 384, 404.

61 I. N. Askerzade, J. Korean Phys. Soc., 2004, 45, 475.

62 I. N. Askerzade, Physics - Uspekhi, 2009, 52, 977.

63 I. Askerzade, Unconventional Superconductors - Anisotropy and Multiband Effects, Springer-Verlag, Berlin Heidelberg, 2012.

64 Y. Kong, O. V. Dolgov and O. Jepsen, et al., Phys. Rev. B: Condens. Matter Mater. Phys., 2001, 64, 1.

65 M. Calandra and F. Mauri, Phys. Rev. B: Condens. Matter Mater. Phys., 2005, 71, 1.

66 D. K. Ferry, S. M. Goodnick and J. Bird, Transport in Nanostructures, Cambridge University Press, Cambridge, Second edn, 2009.

67 J. D. Patterson and B. C. Bailey, Solid-Sate Physics - Introduction to the Theory, Springer Nature, Cham, Switzerland, Third edn, 2018.

68 L. Solymar and D. Walsh, Electrical Properties of Materials, Oxford University Press, Oxford, United Kingdom, Seventh edn, 2004.

69 M. G. Cottam, Dynamical Properties in Nanostructured and Low-Dimensional Materials, IOP Publishing, London, UK, 2015.

70 M. Cardona, Superlattices and Micrsotructures, 1990, 7, 183.

71 V. Davydov, E. Roginskii and Y. Kitaev, et al., Nanomaterials, 2021, 11, 1. 